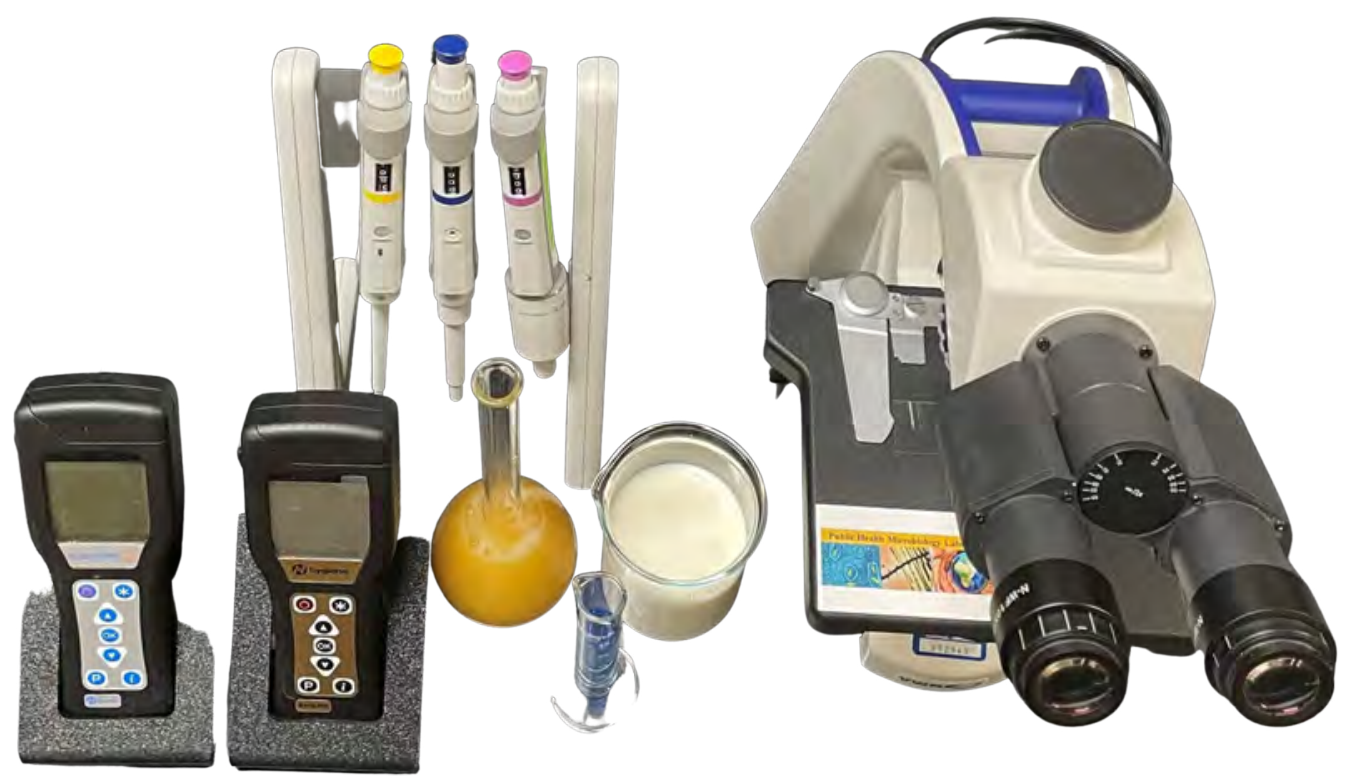

\title{
Sensitivity of Planktonic Cells and Biofilm of Wild-Type and Pressure-Stressed Cronobacter sakazakii and Salmonella enterica Serovars to Sodium Hypochlorite
}

\section{ABSTRAGT}

Aggregate cells are the predominant physiological mode of bacterial proliferation in food processing areas and clinical settings, and microbial biofilms are responsible for the vast majority of all bacterial infections. The current study was conducted to determine whether sodium hypochlorite, validated in the past against planktonic cells, is also efficacious for decontamination of biofilms of wild-type and pressure-stressed nontyphoidal Salmonella enterica serovars and Cronobacter sakazakii. A four-strain mixture of wild-type and pressure-stressed pathogens was used for biofilm formation of up to 14 days on the surface of stainless steel coupons at 7 and $25^{\circ} \mathrm{C}$. Experiments were conducted in two biologically independent repetitions (blocking factors) in a randomized complete block design. Similar biofilm formation trends were observed for wild-type and pressure-stressed phenotypes of $C$. sakazakii. Treatment with sodium hypochlorite was efficacious $(P<0.05)$ only against the planktonic cells and was unable to completely eliminate 1- and 2-week-mature bacterial biofilms of the pathogens.
These findings illustrate that, whereas after a typical sanitization with sodium hypochlorite up to $>3-\log$ reduction of planktonic pathogens is achievable, >6 log of viable pathogens per $\mathrm{cm}^{2}$ of an abiotic surface may survive if bacteria had the opportunity to anchor onto the surface and form biofilm prior to treatment.

\section{INTRODUCTION}

As one of the leading causes of foodborne bacterial infections in American adults, various nontyphoidal Salmonella enterica serovars are an important public health microbiology challenge. Various nontyphoidal serovars of the pathogen are the leading cause of foodborne hospitalizations and death episodes in the United States. In a typical year, over one million Americans are estimated to contract salmonellosis, with 27.2 and $0.5 \%$ hospitalization and death rates, respectively $(10,26)$. This burden is expected to be augmented in the landscape of changing climate because every $1^{\circ} \mathrm{C}$ increase in environmental temperature above $5^{\circ} \mathrm{C}$ could be associated with a 5 to $10 \%$ annual increase in cases of salmonellosis (13). The bacterium is also the 
leading cause of economic concerns associated with the main foodborne pathogens, causing an estimated economic burden of $\$ 3.7$ billion in a typical year in the United States (17) and 32,900 disability-adjusted life years (27). In addition to causing consumer insecurity, the aforementioned economic burden, and public health challenges in the form of illness, hospitalization, and death episodes, nontyphoidal Salmonella serovars are listed in a recent report from the U.S. Centers for Disease Control and Prevention (5) as a "serious threat" for developing antibiotic resistance to medically important classes of antibiotics.

As one of the main foodborne bacterial pathogens of concern in infants, Cronobacter sakazakii similarly has been associated with several national and international outbreaks and is particularly lethal for neonates and preterm infants (16). Since the first reported outbreak of C. sakazakii in 1958, infant formula has been associated with an array of domestic and international episodes of infant morbidity and mortality $(16,21)$.

More than six decades since the initial description of microbial cells in sessile conditions and bacterial biofilms (40), they still remain a major challenge in public health microbiology, health care facilities, and food manufacturing $(9,25,30)$. Microbial biofilms are systematic congregations of microorganisms confined to an extracellular autogenous polymeric matrix, adhering to either biotic or abiotic surfaces $(7,8,15)$. The single or multiple species of microorganisms present in biofilms are, in most cases, protected against environmental stressors, including antimicrobials (18), disinfectants and sanitizers, and immunity of the host (20). This augmentation in resistance causes crucial challenges in decontamination and treatment of microorganisms in health care settings and production facilities (11).

Chlorine-based disinfectants including sodium hypochlorite are one of the most efficacious, cost-effective, and commonly used sanitizing agents in domestic environments, food processing facilities, and health care settings $(24,35)$. Stainless steel is an ideal material for manufacturing surfaces and machinery equipment due to its physicochemical stability and high resistance to corrosion $(19,29)$. Nevertheless, a myriad of examples are available in microbiology and medical literature associated with biofilm formation of pathogenic microorganisms on stainless steel surfaces $(28,29,36)$.

An emerging technology in food manufacturing and the pharmaceutical industry is the use of pressure-based pasteurization for ensuring the safety of an array of products $(1-4,22)$. Although utilization of this emerging and efficacious nonthermal technology is gaining momentum and rapid adaption in the food manufacturing and pharmaceutical industries $(2,4)$, at the current time very limited pieces of information are available regarding the fate and proliferation of pressure-stressed pathogenic microorganisms, i.e., those that are exposed to sublethal hydrostatic pressure (23). For this reason, the purpose of this study is to compare the biofilm formation capability of wild-type and pressure-stressed Salmonella serovars and C. sakazakii at low and ambient temperatures on the surface of stainless steel. The study also investigates the sensitivity of both pathogens (Salmonella serovars and C. sakazakii) and the two phenotypes (wild-type and pressure-stressed) to sodium hypochlorite at various stages of biofilm formation. Currently, Salmonella serovars and C. sakazakii are, respectively, the leading causes of foodborne deaths in American adults (26) and the dominant pathogenic challenge in the manufacturing of infant formula (16).

The current study investigates the biofilm formation of wild-type and pressure-stressed Salmonella serovars and C. sakazakii for up to 3 weeks on the surface of stainless steel coupons and further investigates the sensitivity of planktonic cells and 1-week and 2-week-mature biofilms of both phenotypes of the pathogens to sodium hypochlorite at 7 and $25^{\circ} \mathrm{C}$. Note that the current study explores the sensitivity of the pathogens to the widely used commercial sanitizer under conditions that might not be easy to clean regularly, such as niche and hard-to-reach manufacturing and processing surfaces. Such areas could be a reservoir for mature bacterial biofilms in processing areas.

\section{MATERIALS AND METHODS}

Bacterial culture preparation Strains of Salmonella serovars and C. sakazakii that were used in this study represent two phenotypes of wild-type and pressure-stressed. The cocktail of strains used in this study was selected based on our preliminary work with individual strains (12) and on epidemiological evidence for the selection of serovars with public health importance $(2,3)$. Thus, a four-strain mixture of Salmonella serovars (ATCC 13076 [Enteritidis], 8387 [Montevideo], 6962 [Newport], 14028 [Typhimurium]) and a four-strain mixture of C. sakazakii (ATCC BAA-894, $29544,12868,29004)$ were separately used in the current study. The strains were activated, cultured, subcultured, and purified individually and then were composited prior to inoculation based on the methods articulated in our recent open-access studies $(1-4,22)$. In short, for each strain, a loopful from $-40^{\circ} \mathrm{C}$ frozen glycerol and pathogen stock was transferred into $10 \mathrm{~mL}$ of tryptic soy broth (TSB; Difco, BD, Franklin Lakes, NJ) supplemented with $0.6 \%$ yeast extract (YE) to enhance the recovery of injured cells. Inoculated tubes were then incubated for $24 \mathrm{~h}$ at $37^{\circ} \mathrm{C}$. A $100-\mu \mathrm{L}$ aliquot of this bacterial suspension was streak plated onto the surface of tryptic soy agar (TSA) supplemented with $0.6 \%$ YE and was incubated at $37^{\circ} \mathrm{C}$ for $24 \mathrm{~h}$. These plates of pure cultures were kept at $4^{\circ} \mathrm{C}$ and were used to prepare the bacterial culture for trials.

Prior to the experiments, individual strains were activated by culturing a single colony from the abovementioned plates kept at $4^{\circ} \mathrm{C}$ into $10 \mathrm{~mL}$ of sterile TSB + YE, followed by incubation at $37^{\circ} \mathrm{C}$ for 22 to $24 \mathrm{~h}$. A $100-\mu \mathrm{L}$ aliquot was then subcultured into a different tube containing $10 \mathrm{~mL}$ of TSB 
$+\mathrm{YE}$ and was incubated again at the same temperature and duration. Each strain suspension was harvested by centrifugal force (Rotor FA-45-24-11, model 5424, Eppendorf North America, Hauppauge, NY) at 6,000 rpm $(3,548 \times \mathrm{g}$ for 88 $\mathrm{mm}$ rotor) for $15 \mathrm{~min}$ and was washed with phosphatebuffered saline (VWR International, Radnor, PA) to remove sloughed cell components, excreted secondary metabolites, and growth mediums. Individually subcultured and harvested strains were then composited to prepare inocula of fourstrain mixtures for each pathogen.

\section{Bacterial inoculation}

Formerly used stainless steel coupons (type 304, \#2b finish, [ 1.2 by 5 by $0.3 \mathrm{~cm}$ ]), measured using a digital slide gauge (Marathon Watch Company Ltd., Richmond Hill, Ontario, Canada), were washed and rinsed with detergent, water, 99\% acetone (Fisher Scientific, Fair Lawn, NJ), and $70 \%$ ethanol (Fisher Scientific), to eliminate any residue, and were then sterilized by autoclaving $(11,31)$. Dry, sterilized coupons were spot inoculated with $100 \mu \mathrm{L}$ of wild-type, 10-fold diluted bacterial suspension (target inoculation level of $7.5 \log \mathrm{CFU} / \mathrm{cm}^{2}$ ), and another set of sterilized coupons was inoculated with $100 \mu \mathrm{L}$ of the pressure-stressed suspension (target inoculation level of $7.5 \log \mathrm{CFU} / \mathrm{cm}^{2}$ ). The coupons were stored aseptically for $1 \mathrm{~h}$ at ambient temperature under a biosafety cabinet for attachment of cells onto the surface of coupons. The inoculated coupons were aseptically transferred into $15-\mathrm{mL}$ sterile Eppendorf conical tubes containing $4 \mathrm{~mL}$ of sterile organic milk. This arrangement was done to ensure that coupons were half-submerged, ensuring the existence of air-liquid interface for enhanced development of the bacterial biofilms $(14,37)$. Coupons stayed half-submerged in the food vehicle in an upright tilted position and were incubated statically under aerobic conditions at 7 or $25^{\circ} \mathrm{C}$ for up to 14 days. The time, biofilm formation vehicle, and conditions of attachment for this experiment were selected based on preliminary studies (11). Tubes were incubated at 7 or $25^{\circ} \mathrm{C}$ for both wild-types and pressure-stressed phenotypes, for $0,1,4,7,8,11$, and 14 days.

\section{Formation and decontamination of biofilms}

For investigating the formation of biofilms at 7 and $25^{\circ} \mathrm{C}$, $2 \mathrm{~h}$ after inoculation (day 0 ) and on days $1,4,7,8,11$, and 14 , inoculated coupons were analyzed for microbial populations. Decontamination of the coupons was examined on day 0 (counts of inoculated pathogen on stainless steel coupons prior to biofilm formation, i.e., planktonic cells), day 7 (1-week-mature biofilm), and day 14 (2-week-mature biofilm). Pressure-stressed phenotypes of the pathogens were prepared in the Public Health Microbiology Laboratory of Tennessee State University by exposing the four-strain mixtures of each pathogen to $15,000 \mathrm{lb} / \mathrm{in}^{2}(103.4 \mathrm{MPa})$ of elevated hydrostatic pressure (Barocycler Hub880 Explorer, Pressure BioScience Inc., South Easton, MA) at $25^{\circ} \mathrm{C}$ for
$20 \mathrm{~min}$. This was achieved by controlling the pressure and temperature as explained in our open-access study (2), using HUB Explorer PBI 1.0.8 software (Pressure BioScience Inc.) inside a no-disk PULSE tube (Pressure BioScience Inc.).

The disinfectant used in the current study (Bleach-Rite, catalog no. BRSPRAY128, Fisher Scientific) is registered as a hospital-grade cleaner and contained at least $0.525 \%$ sodium hypochlorite (bleach). The sanitizer was a premix and was used without dilution, i.e., at the manufacturer's maximum registered concentration for disinfection. In addition to receiving a certificate of analysis from the supplier, the free chlorine concentration was verified by measurements using a colorimeter (model HI701, Hanna Instruments, Woonsocket, RI). Ten independent measurements were compared statistically (one-sample $t$-test) with a target concentration of $5,250 \mathrm{ppm}$ (i.e., $0.525 \%)$ and were not statistically $(P \geq 0.05)$ different from this target value. Concentrations of each batch were, in addition, semiquantitatively confirmed to be at least $>5,000$ ppm using a chlorine sanitizer test strip.

For the enumeration of wild-type and pressure-stressed pathogens on surfaces of coupons after inoculation, each side of the coupons was rinsed with $10 \mathrm{~mL}$ of sterilized distilled (total soluble solids $<30 \mathrm{ppm}$ ) water for removal of loosely attached cells (11). For treated samples, after rinsing with sterilized distilled water, $10 \mathrm{~mL}$ of the chlorine-based sanitizer ( $0.525 \%$ sodium hypochlorite) was applied to each side of the coupons. Exposure time of the treatment was 60 $\mathrm{s}$ at $25^{\circ} \mathrm{C}$. Subsequently, each coupon was placed in $30 \mathrm{~mL}$ of Dey-Engley neutralizing broth (Difco, BD) containing 10 sterile glass beads (4-mm diameter). The tubes were then vortexed for $2 \mathrm{~min}(3,200 \mathrm{rpm})$ to detach biofilm cells (11, 32). After this, a 1 -mL aliquot was 10 -fold serially diluted in maximum recovery diluent (Difco, BD) and was spread plated onto TSA + YE. Colonies were manually counted after incubation of plates at $37^{\circ} \mathrm{C}$ for $24 \mathrm{~h}$.

\section{Design and statistical analyses}

The experiments were carried out in two biologically independent repetitions, each considered as a blocking factor in a randomized complete block design. Each biologically independent repetition (each block) consisted of two replications, and each replication further consisted of two microbiological repetitions per sample. Thus, each presented value is the mean of eight independent observations (two blocks, two replicates, two microbiological repetitions). Microbiological data were converted to $\log \mathrm{CFU} / \mathrm{cm}^{2}$, and, after $\log$ transformation of the bacterial counts, the data were analyzed using analysis of variance followed by Tukey-adjusted mean separation for comparison of microbial counts. The statistical analyses were carried out using the general linear models procedure of SAS version 9.4 software (SAS Institute, Inc., Cary, NC) at type 1 error level of 5\% $(\alpha=0.05)$. Experiments involving pathogens at 7 and $25^{\circ} \mathrm{C}$ were conducted independently and, thus, were analyzed and reported separately. 


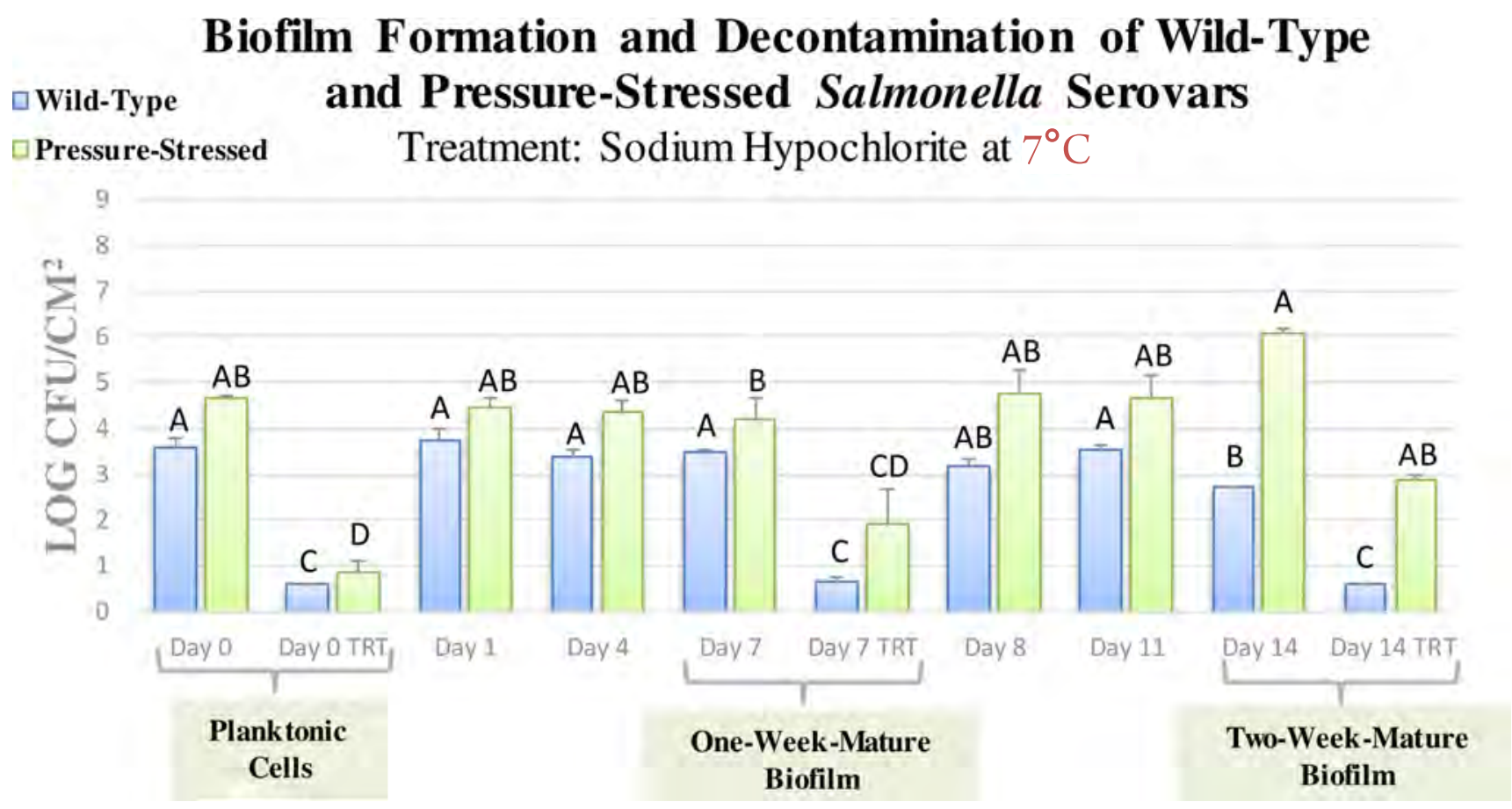

Figure 1. Biofilm formation and decontamination of wild-type and pressure-stressed nontyphoidal Salmonella enterica serovars
at $7^{\circ} \mathrm{C}$. Planktonic Cells = Counts of inoculated pathogen on stainless steel coupons on day 0 prior to biofilm formation.
One-Week-Mature Biofilm = Counts of inoculated pathogen on stainless steel coupons after 7 days of biofilm formation.
Two-Week-Mature Biofilm = Counts of inoculated pathogen on stainless steel coupons after 14 days of biofilm formation.

\section{RESULTS AND DISCUSSION}

Biofilm formation and decontamination of Salmonella serovars at 7 and $25^{\circ} \mathrm{C}$

On day 0 of the trial, counts of wild-type Salmonella serovars were $3.57 \pm 0.2 \log \mathrm{CFU} / \mathrm{cm}^{2}$ at $7^{\circ} \mathrm{C}$. These counts were reduced $(P<0.05)$ to $<0.58 \pm 0.0 \log \mathrm{CFU} / \mathrm{cm}^{2}$ on day 0 , after the treatment with sodium hypochlorite at the highest concentration recommended by the manufacturer $(0.525 \%$ sodium hypochlorite) for disinfection of abiotic surfaces. This reduction of at least $3 \log$ (i.e., 99.9\%) of planktonic cells (counts of inoculated pathogen on stainless steel coupons on day 0 prior to biofilm formation) is in harmony with the manufacturer's validation studies and existing peer-reviewed literature (11). Thus, this indicates that the intervention is capable of eliminating biologically significant amounts of bacterial pathogens in the planktonic stage from stainless steel surfaces (Fig. 1). The wild-type pathogen at this low temperature was not able to multiply $(P \geq 0.05)$. Biofilm counts of days $1,4,7,8$, and 11 were $3.74 \pm 0.2$, $3.36 \pm 0.2,3.48 \pm 0.0,3.20 \pm 0.1$, and $3.51 \pm 0.1 \log \mathrm{CFU} /$ $\mathrm{cm}^{2}$, respectively (Fig. 1). One-week and 2-week-mature biofilms had comparable sensitivity to the sanitizer relative to planktonic cells (counts on day 0 ) at this temperature. The counts of 1 -week and 2-week-mature biofilms at $7^{\circ} \mathrm{C}$ were reduced $(P<0.05)$ by 2.82 and 2.13 log, respectively (Fig. 1$)$. The pressure-stressed phenotype of the pathogen exhibited similar susceptibility to the sanitizer. Counts of the pressurestressed Salmonella serovars on days $0,1,4,7$, and 14 were $4.63 \pm 0.1,4.46 \pm 0.2,4.36 \pm 0.2,4.21 \pm 0.4$, and $6.09 \pm 0.1$ $\log \mathrm{CFU} / \mathrm{cm}^{2}$, respectively (Fig. 1).

However, note that after the treatment with sodium hypo-chlorite on days 7 and 14 and at $7^{\circ} \mathrm{C}$, although there were 2.82- and 2.13-log reductions $(P<0.05)$ of the pathogen, the treatment left behind biologically appreciable amounts of pathogens on the treatment surface. Bacterial loads of the stainless steel coupons were $1.90 \pm 0.1$ and $2.89 \pm 0.1 \log \mathrm{CFU} / \mathrm{cm}^{2}$ after the treatment, respectively (Fig. 1). Note that $2 \log \mathrm{CFU}$ of Salmonella serovars is equivalent to 100 cells of the pathogen per square centimeter of stainless steel, and previous literature indicates that 10 to 1,000 cells of nontyphoidal Salmonella are capable of causing health complications and mortality in humans, if ingested (34). The ability of Salmonella serovars to maintain sessile communities at this low temperature and the similarity between biofilm formation of wild-type and pressure-stressed phenotypes observed in this study is in harmony with previous literature. Similarly, a new study conducted in sterilized surface water observed comparable biofilm formation of these phenotypes at $5^{\circ} \mathrm{C}(23)$.

As expected, Salmonella serovars exhibited considerably higher multiplication and biofilm formation at 25 compared to $7^{\circ} \mathrm{C}$ (Fig. 2). This finding is in harmony with a 1-month trial in which multiplication and biofilm formation of 


\section{Biofilm Formation and Decontamination of Wild-Type \\ $\square$ Wild-Type and Pressure-Stressed Salmonella Serovars}

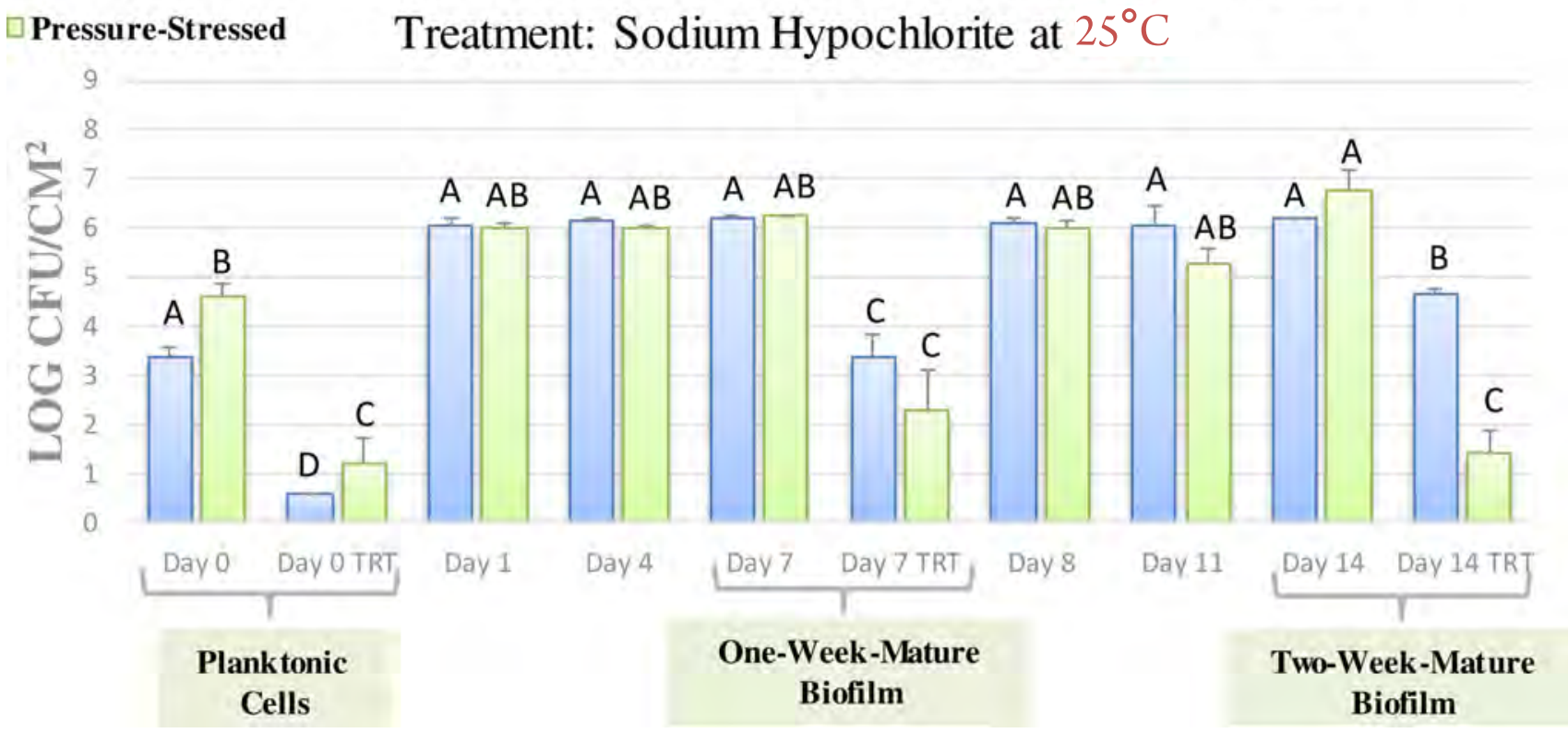

Figure 2. Biofilm formation and decontamination of wild-type and pressure-stressed nontyphoidal Salmonella enterica at $25^{\circ} \mathrm{C}$. Planktonic Cells $=$ Counts of inoculated pathogen on stainless steel coupons on day 0 prior to biofilm formation. One-Week-Mature Biofilm = Counts of inoculated pathogen on stainless steel coupons after 7 days of biofilm formation. Two-Week-Mature Biofilm = Counts of inoculated pathogen on stainless steel coupons after 14 days of biofilm formation.

nontyphoidal Salmonella serovars were compared at 5, 25 , and $37^{\circ} \mathrm{C}$ (23). At this temperature, pressure-stressed and wild-type phenotypes exhibited comparable biofilm formation and sensitivity to sanitizer at the vast majority of tested time intervals. On day 0 , counts of wild-type and pressure-stressed pathogens were $3.39 \pm 0.2$ and 4.60 $\pm 0.2 \log \mathrm{CFU} / \mathrm{cm}^{2}$, respectively (Fig. 2). These counts were reduced by $>2.81$ and $3.36 \log$ to $<0.58 \pm 0.0$ and 1.23 $\pm 0.5 \log \mathrm{CFU} / \mathrm{cm}^{2}$, respectively, after the abovementioned treatment (Fig. 2). These results indicate that the widely used sanitizer is microbiologically efficacious for at least $99 \%$ reduction of the pathogen, when tested against planktonic cells, and that it leaves behind less than 10 to $100 \mathrm{CFU/}$ $\mathrm{cm}^{2}$ of the pathogen on steel surfaces after the treatment. This efficacy of the sanitizer, at the highest concentration recommended by the manufacturer, was diminished when tested against 1 - and 2-week-mature biofilms. On day 7 of biofilm formation, counts of the wild-type and pressurestressed cells were $6.20 \pm 0.1$ and $6.23 \pm 0.0 \log \mathrm{CFU} / \mathrm{cm}^{2}$, respectively (Fig. 2). After the abovementioned treatment with sodium hypochlorite, the counts were reduced to $3.38 \pm 0.4$ and $2.30 \pm 0.8 \log \mathrm{CFU} / \mathrm{cm}^{2}$, respectively (Fig. 2). Although reductions of 2.81 and $3.93 \mathrm{log} \mathrm{CFU} / \mathrm{cm}^{2}$ were observed after decontamination of the 1-week mature biofilm, there were biologically important amounts of pathogen left on the surface of stainless steel after treatment.
This indicates that the commonly used sanitizer is microbiologically efficacious against planktonic cells of the pathogen but is not capable of complete elimination of 1-week-mature biofilm. Thus, our study highlights the need for validation studies against bacterial biofilms, in addition to planktonic cells, to ensure that sanitization programs currently in place in food and health care facilities are capable of eliminating both phenotypes. These findings were also observed for 2-week-mature biofilms (Fig. 2) and are of particular importance for ensuring the hygiene of niche and hard-to-reach surfaces.

Previous research has shown similar outcomes about the decontamination efficacy of sodium hypochlorite and its diminished capability against bacterial biofilm. As an example, one study showed that 3-day-old Salmonella biofilm is more resistant than planktonic cells are to this sanitizer (39). Similarly, sodium hypochlorite was unable to completely eliminate 48 -h and 168-h-mature Salmonella biofilms (6). Note that studies comparing the sensitivity of 3-day, 5-day, and 7-day-mature biofilms of Salmonella exposed to sodium hypochlorite indicate that these three ages of biofilm were not different from each other in regard to resistance to the disinfectant (38). Thus, our study results could be relevant not only to 1 - and 2-week-mature biofilms but also to biofilm masses of less than 1 week of age. 


\title{
Biofilm Formation and Decontamination of Wild-Type and Pressure-Stressed Cronobacter sakazakii
}

\section{$\square$ Wild-Type \\ $\square$ Pressure-Stressed}

\author{
Treatment: Sodium Hypochlorite at $7^{\circ} \mathrm{C}$
}

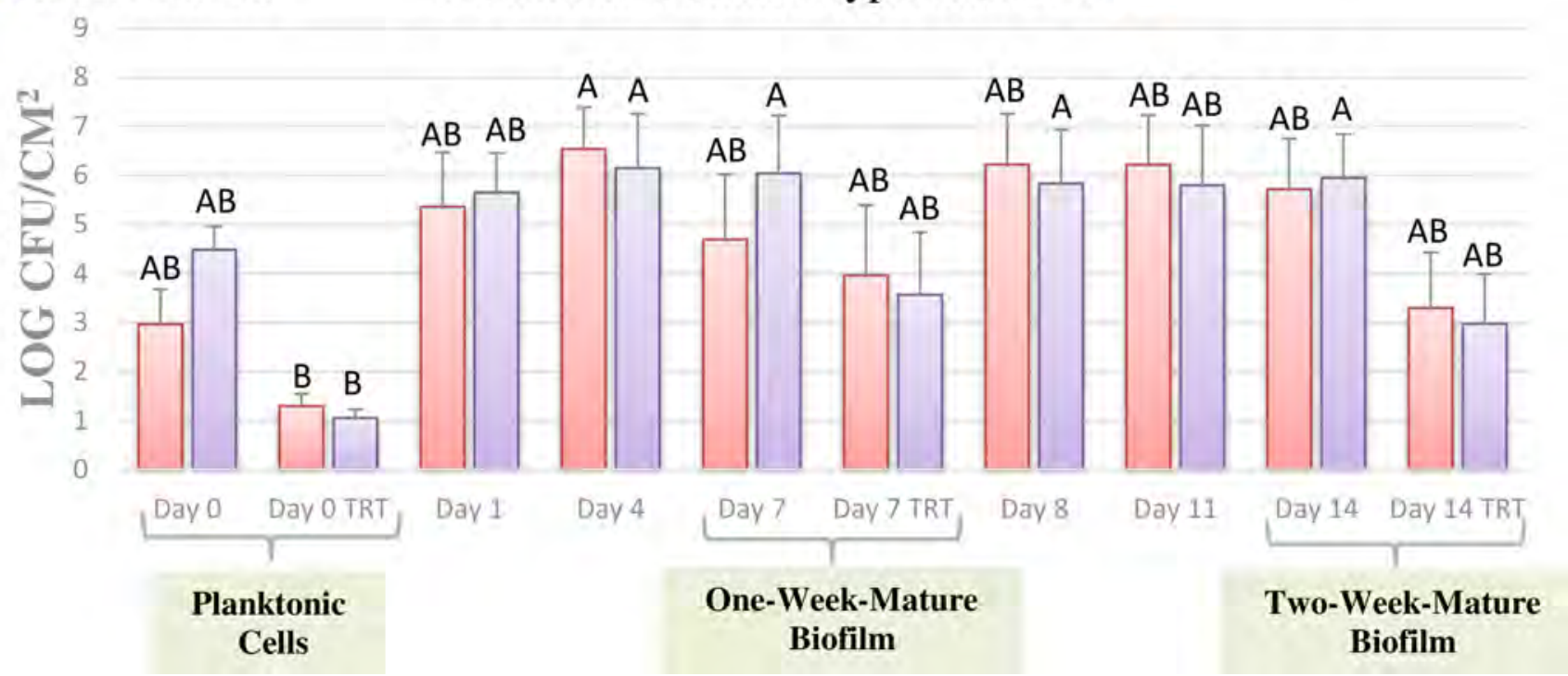

\begin{abstract}
Figure 3. Biofilm formation and decontamination of wild-type and pressure-stressed Cronobacter sakazakii at $7^{\circ} \mathrm{C}$. Planktonic Cells = Counts of inoculated pathogen on stainless steel coupons on day 0 prior to biofilm formation. One-Week-Mature Biofilm = Counts of inoculated pathogen on stainless steel coupons after 7 days of biofilm formation. Two-Week-Mature Biofilm = Counts of inoculated pathogen on stainless steel coupons after 14 days of biofilm formation.
\end{abstract}

Biofilm formation and decontamination of C. sakazakii serovars at 7 and $25^{\circ} \mathrm{C}$

Pathogenic species of Cronobacter are primarily associated with contamination of infant formula in manufacturing and during preparation in domestic environments and in health care facilities. During manufacturing of powdered infant formula, heat-sensitive nutrients of the product are typically added after heat treatment, to meet the strict regulatory requirement for nutritional value of the product. This practice is the common route associated with crosscontamination of the product after thermal processing and before final packaging (16). Similarly, during preparation of the formula, pathogenic species of Cronobacter on abiotic preparation surfaces could cross-contaminate the product. A review of the literature exhibits an array of domestic and international outbreaks associated with C. sakazakii and infant morbidity and mortality, including two outbreaks in Memphis and Knoxville in the state of Tennessee (16).

Although, under the condition of our experiments at $7^{\circ} \mathrm{C}$, C. sakazakii counts did not $(P \geq 0.05)$ appreciably increase during the trials, the bacterium was able to form and maintain a biofilm mass on stainless steel (Fig. 3). The counts of wild-type C. sakazakii were $2.96 \pm 0.7 \mathrm{log} \mathrm{CFU} / \mathrm{cm}^{2}$ on day 0 of the experiment. These counts were $4.69 \pm 1.3$ and $5.72 \pm 1.0 \log \mathrm{CFU} / \mathrm{cm}^{2}$ on days 7 and 14, respectively (Fig. 3). Counts of the pressure-stressed phenotypes on days
0,7 , and 14 were $4.48 \pm 0.5,6.04 \pm 1.2$, and 5.95 $\pm 1.0 \mathrm{log} \mathrm{CFU} / \mathrm{cm}^{2}$, respectively (Fig. 3). On day 0 , when the antimicrobial intervention was used against the planktonic cells, the vast majority of inoculated pathogen cells were decontaminated from the surface of stainless steel. Treatments on this day resulted in 1.67- and 3.43-log reductions of wild-type and pressure-stressed pathogens and left behind $1.30 \pm 0.2$ and $1.06 \pm 0.2 \log \mathrm{CFU} / \mathrm{cm}^{2}$ on the abiotic surface, respectively (Fig. 3). The same treatment, when tested against 1-week-mature biofilm, exhibited appreciably less efficacy. Wild-type counts of the pathogen on day 7 were $4.69 \pm 1.3$ and $3.97 \pm 1.3 \log \mathrm{CFU} / \mathrm{cm}^{2}$ before and after the treatment, respectively (Fig. 3). Similarly, counts of pressure-stressed pathogens were $6.04 \pm 1.2$ and $3.57 \pm 1.3$ $\log \mathrm{CFU} / \mathrm{cm}^{2}$ before and after the treatment, respectively (Fig. 3). Whereas treatments against 2-week-mature biofilm resulted in 2.41- and 2.98-log reductions of wild-type and pressure-stressed phenotypes of C. sakazakii, there were a considerable number of survivors after treatment, which illustrates that the antimicrobial intervention is not able to completely eliminate 2 -week-mature biofilms. After the treatment, counts of 2-week-mature biofilms of the pathogen were $3.30 \pm 1.1$ and $3.97 \pm 1.0 \log \mathrm{CFU} / \mathrm{cm}^{2}$ for wild-type and pressure-stressed phenotypes, respectively (Fig. 3).

These results illustrate that a successful sanitization program requires optimization and validation studies to 


\section{Biofilm Formation and Decontamination of Wild-Type and Pressure-Stressed Cronobacter sakazakii}

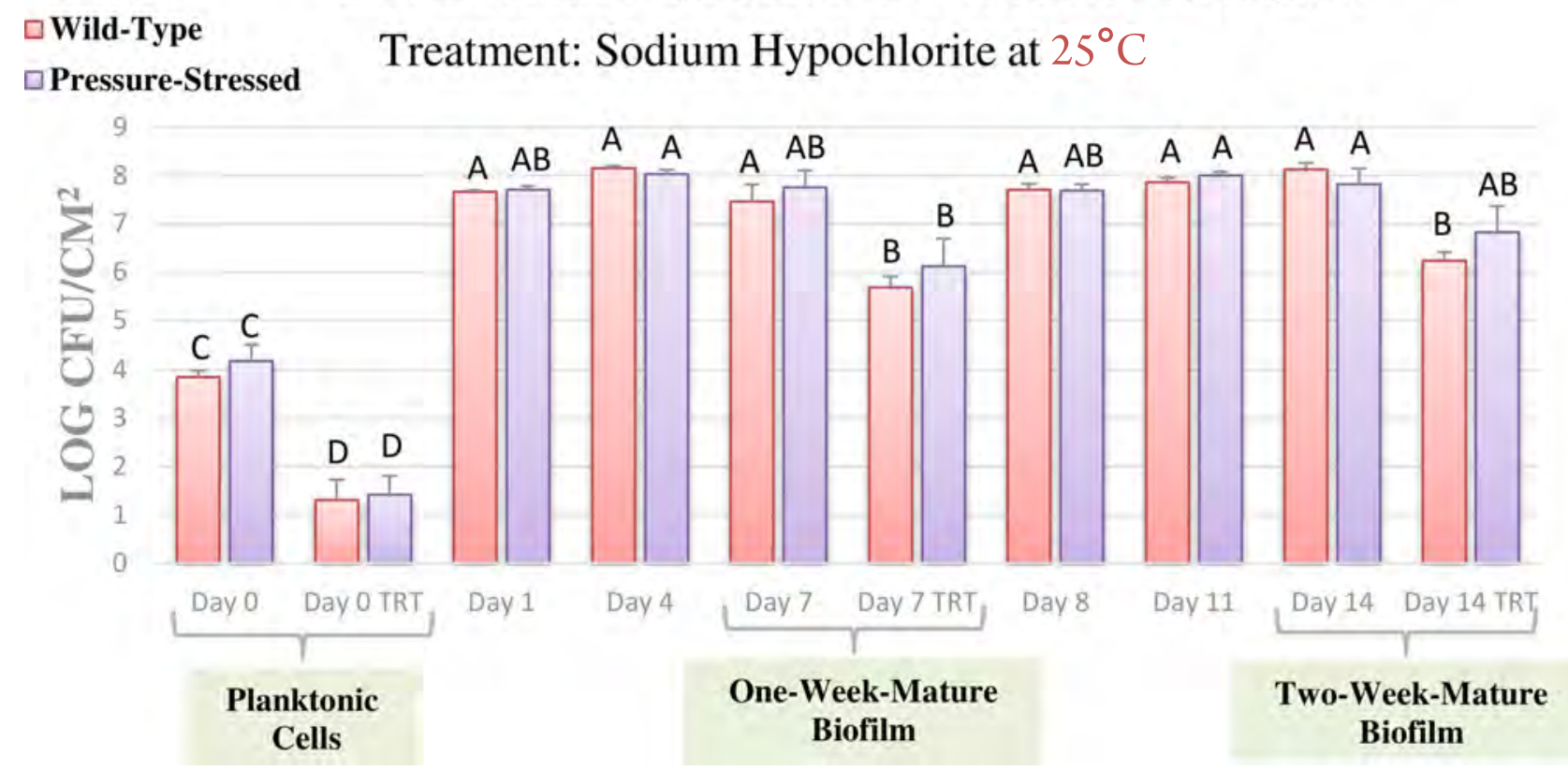

Figure 4. Biofilm formation and decontamination of wild-type and pressure-stressed Cronobacter sakazakii at $25^{\circ} \mathrm{C}$.

Planktonic Cells = Counts of inoculated pathogen on stainless steel coupons on day 0 prior to biofilm formation. One-Week-Mature Biofilm = Counts of inoculated pathogen on stainless steel coupon after 7 days of biofilm formation. Two-Week-Mature Biofilm = Counts of inoculated pathogen on stainless steel coupons after 14 days of biofilm formation.

ensure that an antimicrobial intervention is microbiologically validated against planktonic and sessile cells of the pathogen to ensure the safety of the product in manufacturing and care setting environments. A previous study also reported the low efficacy of commercial sanitizers against 1-weekmature biofilms: treatments based on acid and quaternary ammonium compounds showed efficacy against planktonic cells but were unable to completely eliminate sessile cells anchored to the surface of stainless steel coupons (11).

Pathogen multiplication and biofilm formation, as expected, were considerably more pronounced at $25^{\circ} \mathrm{C}$ (Fig. 4), raising even higher concerns about the efficacy of a very common sanitizer against bacterial biofilms when used at ambient temperature. On day 0 , counts of the wild-type pathogen were $3.85 \pm 0.1 \log \mathrm{CFU} / \mathrm{cm}^{2}$. These counts increased to $7.66 \pm 0.0,8.16 \pm 0.1,7.47 \pm 0.4,7.86 \pm 0.1$, and $8.12 \pm 0.1 \log \mathrm{CFU} / \mathrm{cm}^{2}$ on days $1,4,7,11$, and 14 , at $25^{\circ} \mathrm{C}$, respectively (Fig. 4). Corresponding counts of the pressurestressed phenotype were similar, $7.71 \pm 0.0,8.03 \pm 0.1,7.75$ $\pm 0.4,8.00 \pm 0.1$, and $7.82 \pm 0.1 \log \mathrm{CFU} / \mathrm{cm}^{2}$ (Fig. 4). At this temperature, treatment with sodium hypochlorite on day 0 was able to bring down $(P<0.05)$

C. sakazakii counts of both phenotypes close to the detection limit. Treatment was able to cause 2.54- and 2.77log reductions of wild-type and pressure-stressed phenotypes and left behind $1.31 \pm 0.4$ and $1.42 \pm 0.4 \log \mathrm{CFU} / \mathrm{cm}^{2}$ of the pathogen, respectively (Fig. 4). On day 7, for both phenotypes, the treatment was effective $(P<0.05)$ for less than 2-log reductions of both phenotypes. After the treatment on day 7, 5.69 \pm 0.2 and $6.13 \pm 0.6 \log \mathrm{CFU} /$ $\mathrm{cm}^{2}$ of the pathogen were still present on the surface of stainless steel coupons (Fig. 4). This indicates that if C. sakazakii contamination occurs on an abiotic surface and has sufficient time to form mature biofilms, a typical sanitizer very common in health care and food manufacturing facilities may not completely eliminate the pathogen and a validated cleaning and sanitation is required to ensure complete removal of C. sakazakii biofilms.

The lack of efficacy of the tested sanitizer was even more pronounced in the decontamination trials against the 2-weekmature biofilms. The treatment led to 1.89- and 1.00-log reductions of wild-type and pressure-stressed biofilm cells and left behind $6.24 \pm 0.2$ and $6.83 \pm 0.5 \log \mathrm{CFU} / \mathrm{cm}^{2}$ of the 2-week-mature sessile cells of the pathogen, respectively (Fig. 4). These findings illustrate that, even after a very common and typical sanitization program, in excess of $1,000,000$ cells (i.e., $>6 \log$ ) of viable pathogenic C. sakazakii per $\mathrm{cm}^{2}$ of an abiotic surface may exist if the pathogen had the opportunity to anchor to the surface and form sessile communities prior to the treatment. Although decontamination of C. sakazakii biofilms with sodium hypochlorite has been only modestly investigated in the 
literature and no similar design to the current study was located, the application of other antimicrobials such as benzalkonium chloride, peroxyacetic acid, and chlorine dioxide, even for durations as long as $15 \mathrm{~min}$, have been reported to leave behind as much as $72 \%$ of the 6-day-old biofilms of this pathogen (33).

\section{CONCLUSIONS}

Under the conditions of our experiment, we observed that a treatment with sodium hypochlorite, at the concentration commonly used in private industry and health care facilities, could be very efficacious for removal of planktonic cells from stainless steel surfaces. However, the same treatment is less efficacious against 1-week and 2-week-mature biofilms of nontyphoidal Salmonella serovars and C. sakazakii. The inability of this common sanitizer to completely eliminate these biofilms was even more pronounced when sessile cells were formed at higher temperatures. We additionally observed that pressure-stressed (i.e., those surviving a sublethal pressure-based treatment) Salmonella serovars and C. sakazakii pathogens could not only proliferate and form biofilms but also exhibit comparable resistance to the antimicrobial treatment relative to their immediate wild-type phenotypes.
Thus, our results highlight the need for microbiological validation studies to consider both planktonic and biofilm phenotypes of pathogens. This approach could ensure external validity of a commercial sanitization program and its efficacy in health care and manufacturing facilities. These findings are of importance for validation of sanitation standard operating procedures to meet regulatory requirements of legislations such as hazard analysis and critical control point and the U.S. Food Safety Modernization Act, particularly for ensuring the microbiological safety of surfaces under conditions that might not be easy to clean regularly, such as niche and hard-to-reach manufacturing and processing areas.

\section{ACKNOWLEDGMENTS}

Technical contributions and administrative support of the members of the Public Health Microbiology laboratory are sincerely appreciated by the authors. Financial support in part from the National Institute of Food and Agriculture of the U.S. Department of Agriculture (projects 2017-07534; 2017-07975; 2017-06088) and Pressure BioScience Inc. are acknowledged gratefully by the corresponding author.

\section{REFERENCES}

1. Allison, A., S. Chowdhury, and A. C. Fouladkhah. 2018. Synergism of mild heat and high-pressure pasteurization against Listeria monocytogenes and natural microflora in phosphate-buffered saline and raw milk. Microorganisms 6:102-114.

2. Allison, A., E. Daniels, S. Chowdhury, and A. C. Fouladkhah. 2018. Effects of elevated hydrostatic pressure against mesophilic background microflora and habituated Salmonella serovars in orange juice. Microorganisms 6:23-35.

3. Allison, A., and A. C. Fouladkhah. 2018. Sensitivity of Salmonella serovars and natural microflora to high-pressure pasteurization: open access data for risk assessment and practitioners. Data Brief 21:480-484.

4. Aras, S., M. N. Kabir, S. Chowdhury, and A. C. Fouladkhah. 2020. Augmenting the pressure-based pasteurization of Listeria monocytogenes by synergism with nisin and mild heat. Int. J. Environ. Res. Publ. Health $17: 563-566$.

5. Centers for Disease Control and Prevention 2019. Antibiotic resistance threats in the United States. Available at: https://www. cdc.gov/drugresistance/pdf/threatsreport/2019-ar-threats-report-508.pdf. Accessed 19 September 2020
6. Corcoran, M., D. Morris, N. De Lappe, J. O'Connor, P. Lalor, P. Dockery, and M. Cormican. 2014. Commonly used disinfectants fail to eradicate Salmonella enterica biofilms from food contact surface materials. Applied Environ. Microbiol. 80:1507-1514.

7. Costerton, J. W., P. S. Stewart, and E. P. Greenberg. 1999. Bacterial biofilms: a common cause of persistent infections. Science 284:1318-1322.

8. Donlan, R. M., and J. W. Costerton. 2002 Biofilms: survival mechanisms of clinically relevant microorganisms. Clin. Microbiol. Rev. 15:167-193.

9. Flint, S. H., P. J. Bremer, and J. D. Brooks. 1997. Biofilms in dairy manufacturing plantdescription, current concerns and methods of control. Biofouling 11:81-97.

10. Fouladkhah, A. 2017. The need for evidence-based outreach in the current food safety regulatory landscape. J. Ext. 55 (2). (Commentary.)

11. Fouladkhah, A., I. Geornaras, and J. N. Sofos. 2013. Biofilm formation of O157 and non-O157 Shiga toxin-producing Escherichia coli and multidrug-resistant and susceptible Salmonella Typhimurium and Newport and their inactivation by sanitizers. J. Food Sci. 78:M880-M886.
12. Fouladkhah, A., I., Geornaras, H. Yang, and J. N. Sofos. 2013. Lactic acid resistance of Shiga toxin-producing Escherichia coli and multidrug-resistant and susceptible Salmonella Typhimurium and Salmonella Newport in meat homogenate. Food Microbiol. 36:260-266.

13. Fouladkhah, A., B. Thompson, and J. S. Camp. 2019. Safety of food and water supplies in the landscape of changing climate. Microorganisms 7:469-474.

14. Giaouris, E. D., and G. J. E. Nychas. 2006. The adherence of Salmonella Enteritidis PT4 to stainless steel: the importance of the airliquid interface and nutrient availability. Food Microbiol. 23:747-752.

15. Hall-Stoodley, L., F. Z. Hu, A. Gieseke, L. Nistico, D. Nguyen, J. Hayes, M. Forbes, D. P. Greenberg, B. Dice, A. Burrows, and P. A. Wackym. 2006. Direct detection of bacterial biofilms on the middle-ear mucosa of children with chronic otitis media. JAMA (J. Am. Med. Assoc.) 296:202-211.

16. Henry, M., and A. Fouladkhah. 2019. Outbreak history, biofilm formation, and preventive measures for control of Cronobacter sakazakii in infant formula and infant care settings. Microorganisms 7:77-99. 
17. Hoffman, S., B. MaCulloch, and M. Batz. 2015. Economic burden of major foodborne illnesses acquired in the United States. EIB-140. U.S. Department of Agriculture, Economic Research Service. Available at: https://www.ers.usda. gov/publications/pub-details $/$ ?pubid $=43987$. Accessed 19 September 2020.

18. Hoiby, N., T. Bjarnsholt, M. Givskov, S. Molin, and O. Ciofu. 2010. Antibiotic resistance of bacterial biofilms. Int. J. Antimicrob. Agents 35:322-332.

19. Holah, J. T., and R. H. Thorpe. 1990. Cleanability in relation to bacterial retention on unused and abraded domestic sink materials. J. Appl. Bacteriol. 69:599-608.

20. Jensen, P. O., M. Givskov, T. Bjarnsholt, and C. Moser. 2010. The immune system vs. Pseudomonas aeruginosa biofilms. FEMS Immunol. Med. Microbiol. 59:292-305.

21. Jones, G., M. P. de la Gandara, L. HerreraLeon, S. Herrera-Leon, C. V. Martinez, R. Hureaux-Roy, Y. Abdallah, A. Nisavanh, L. Fabre, C. Renaudat, and J. Mossong. 2019. Outbreak of Salmonella enterica serotype Poona in infants linked to persistent Salmonella contamination in an infant formula manufacturing facility, France, August 2018 to February 2019. Euro Surveill. 24:1-6.

22. Kabir, M. N., S. Aras, A. Allison, J. Adhikari, S. Chowdhury, and A. F. Fouladkhah. 2019. Interactions of carvacrol, caprylic acid, habituation, and mild heat for pressurebased inactivation of $\mathrm{O} 157$ and non-O157 serogroups of Shiga toxin-producing Escherichia coli in acidic environment. Microorganisms 7:145-160.

23. Kabir, M. N., S. Aras, S. Wadood, S. Chowdhury, and A. C. Fouladkhah. 2020. Fate and biofilm formation of wild-type and pressure-stressed pathogens of public health concern in surface water and on abiotic surfaces. Microorganisms 8:408-422.
24. Liu, C., J. Duan, and Y. S. Su. 2006. Effects of electrolyzed oxidizing water on reducing Listeria monocytogenes contamination on seafood processing surfaces. Int. J. Food Microbiol. 106:248-253.

25. Maukonen, J., J. Matto, G. Wirtanen, L. Raaska, T. Mattila-Sandholm, and M. Saarela. 2003. Methodologies for the characterization of microbes in industrial environments: a review. J. Indust. Microbiol. Biotechnol. 30:327-356.

26. Scallan, E., R. M. Hoekstra, F. J. Angulo, R. V. Tauxe, M. A. Widdowson, S. L. Roy, J. L. Jones, and P. M. Griffin. 2011. Foodborne illness acquired in the United Statesmajor pathogens. Emerg. Infect. Dis. 17:7-16.

27. Scallan, E., R. M. Hoekstra, B. E. Mahon, T. F. Jones, and P. M. Griffin. 2015. An assessment of the human health impact of seven leading foodborne pathogens in the United States using disability adjusted life years. Epidemiol. Infect. 143:2795-2804.

28. Sharma, M., and S. K. Anand. 2002. Characterization of constitutive microflora of biofilms in dairy processing lines. Food Microbiol. 19:627-636.

29. Shi, X., and X. Zhu. 2009. Biofilm formation and food safety in food industries. Trends Food Sci. Technol. 20:407-413.

30. Sihorkar, V., and S. P. Vyas. 2001. Biofilm consortia on biomedical and biological surfaces: delivery and targeting strategies. Pharm. Res. 18:1247-1254.

31. Simpson Beauchamp, C., D. Dourou, I. Geornaras, Y. Yoon, J. A. Scanga, K. E. Belk, G. C. Smith, G. J. E. Nychas, and J. N. Sofos. 2012. Transfer, attachment, and formation of biofilms by Escherichia coli O157: $\mathrm{H} 7$ on meat-contact surface materials. J. Food Sci. 77:M343-M347.

32. Stopforth, J. D., J. Samelis, J. N. Sofos, P. A. Kendall, and G. C. Smith. 2002. Biofilm formation by acid-adapted and nonadapted Listeria monocytogenes in fresh beef decontamination washings and its subsequent inactivation with sanitizers. J. Food Prot. 65:1717-1727.
33. Torlak, E., M. Gokmen, and S. Aydemir. 2015. Efficacy of disinfectants against Cronobacter biofilm on plastic surfaces. Qual. Assur. Saf. Crop. 7:819-823.

34. U.S. Food and Drug Administration. 2017. Bad bug book: handbook of foodborne pathogenic microorganisms and natural toxins. Available at: https://www.fda.gov/ files/food/published/Bad-Bug-Book-2ndEdition-\%28PDF\%29.pdf. Accessed 19 September 2020.

35. U.S. Food and Drug Administration. 2019. Indirect food additives: adjuvants, production aids, and sanitizers, final rule. 21 CFR 178.3570. Fed. Regist. 84:26746-26747.

36. Vestby, L. K., T. Moretro, S. Langsrud, E. Heir, and L. L. Nesse. 2009. Biofilm forming abilities of Salmonella are correlated with persistence in fish meal-and feed factories. BMC Vet. Res. 5:1-6.

37. Wijman, J. G. E., P. P. L. A. de Leeuw, R. Moezelaar, M. H. Zwietering, and T. Abee. 2007. Air-liquid interface biofilms of Bacillus cereus: formation, sporulation, and dispersion. Applied Environ. Microbiol. 73:1481-1488.

38. Wong, H. S., K. M. Townsend, S. G. Fenwick, G. Maker, R. D. Trengove, and R. M. O' Handley. 2010. Comparative susceptibility of Salmonella Typhimurium biofilms of different ages to disinfectants. Biofouling 26:859-864.

39. Wong, H. S., K. M. Townsend, S. G. Fenwick, R. D. Trengove, and R. M. O'Handley. 2010. Comparative susceptibility of planktonic and 3-day-old Salmonella Typhimurium biofilms to disinfectants. J. Appl. Microbiol. 108:2222-2228.

40. Zobell, C. E. 1943. The effect of solid surfaces upon bacterial activity. J. Bacteriol. 46:39-56. 